\title{
Strong purified protein derivative responses are associated with poor mycobacterium inhibition in latent TB
}

\author{
J.S.L. Kang*, A. Cherian" ${ }^{\#}$ S.H. Gan\#, T.H. Lee ${ }^{\star}$, K.C. Lee ${ }^{\Uparrow}$, C.B.E. Chee ${ }^{\#}$, \\ T.M. Doherty ${ }^{+}$, Y.T. Wang ${ }^{\#}$ and G.T. Seah*
}

ABSTRACT: The tuberculin skin test (TST) using purified protein derivative (PPD) of Mycobacterium tuberculosis is traditionally used to diagnose latent tuberculosis (TB) infection (LTBI). However, LTBI diagnosis by peripheral blood mononuclear cell (PBMC) interferon (IFN)- $\gamma$ responses to $M$. tuberculosis-specific antigens, early secreted antigenic target 6 kDa (ESAT-6) and culture filtrate protein (CFP)-10 has greater specificity. We investigated the difference in antimycobacterium cellular immunity in TB contacts who were strong TST reactors but nonresponsive to the ESAT-6/CFP-10 assay compared with those with concordant results.

Healthy TB contacts were tested using the above two assays and mycobacterium survival was measured after co-culture of infected macrophages with their PBMCs.

Whether PPD reactivity was tested by TST or by PBMC-specific IFN- $\gamma$ responses, strongly PPDreactive TB contacts without ESAT-6/CFP-10 responsiveness showed significantly better mycobacterium inhibition activity than ESAT-6/CFP-10-responsive TB contacts with the same PPD reactivity. In the former group, stronger PPD reactivity was associated with improved mycobacterium killing, whereas ESAT-6/CFP-10 responders showed the opposite result.

PPD-reactive ESAT-6/CFP-10-nonresponsive TB contacts in our population may have had protective immunity related to prior mycobacterium exposure. ESAT-6/CFP10-responsive TB contacts are more likely to have LTBI and, in this group, strong PPD reactivity may paradoxically be associated with poor mycobactericidal activity.

KEYWORDS: Cytokine production, immunity, tuberculosis, tuberculin skin test

$\mathbf{T}$ he tuberculin skin test (TST), representing delayed-type hypersensitivity to purified protein derivative (PPD) of Mycobacterium tuberculosis, is often used for screening tuberculosis (TB)-exposed contacts. Strong TST reactivity is usually interpreted as indicating latent TB infection (LTBI). However, PPD responses may be due to past Mycobacterium bovis bacille CalmetteGuérin (BCG) vaccinations [1] or immune exposure to environmental mycobacteria [2], as various Mycobacterium species have many genes in common. Thus, using the TST for LTBI diagnosis gives poor specificity in areas where the climate favours environmental mycobacterium proliferation [3] and where a routine BCG vaccination and revaccination programme exists.

Early secreted antigenic target $6 \mathrm{kDa}$ (ESAT-6) and culture filtrate protein (CFP)-10 are immunogenic ТВ proteins encoded by genes absent in all BCG strains and most environmental mycobacteria [4]. The use of in vitro interferon (IFN)- $\gamma$ release assays with ESAT-6/CPF-10 as $M$. tuberculosis-specific antigens therefore avoids cross-reactivity in BCG-vaccinated subjects, thereby overcoming some limitations of the TST. The utility of these assays has been extensively reviewed $[5,6]$. Numerous human studies worldwide have shown that ESAT-6/CFP-10 is more specific than PPD in detecting LTBI $[7,8]$. Although some studies suggest that the sensitivity of ESAT-based assays is lower than the TST [9], later studies show otherwise [10]. Moderately good agreement $(60-80 \%)$ and positive correlation between the TST and various IFN- $\gamma$-based assays has been reported [6, 11]. However, the immune status of TB contacts with discordant results in PPD- and ESAT-based tests has yet to be fully resolved.

\section{AFFLLIATIONS}

${ }^{*}$ Dept of Microbiology and Immunology Programme, Yong Loo Lin School of Medicine, National University of Singapore,

\#Tuberculosis Control Unit, Tan Tock Seng Hospital, and

"Clifford Dispensary, Singapore. ${ }^{+}$Dept of Infectious Disease

Immunology, Statens Serum Institute, Copenhagen, Denmark.

\section{CORRESPONDENCE}

G.T. Seah

Dept of Microbiology

National University of Singapore MD4

5 Science Drive 2

Singapore 117597

Singapore

E-mail: micsgt@nus.edu.sg

Received:

April 162009

Accepted after revision:

Nov 222009

First published online:

Dec 082009 
The lack of IFN- $\gamma$ activity severely compromises host immunity in human TB [12]. However, the protective implications of high TB-specific IFN- $\gamma$ levels (e.g. IFN- $\gamma$ responses of PPDstimulated lymphocytes) are ambiguous, as IFN- $\gamma$ expression does not directly correlate with protective outcomes when TBexposed contacts are followed up for TB reactivation [13]. We postulate that this could be because people with strong PPDspecific responses attributable to different clinical situations (e.g. active TB infection, LTBI, BCG vaccination or exposure to environmental mycobacteria) may have different levels of protective immunity. This may also underlie discrepancies in outcomes of ESAT versus PPD response assays.

Mycobacterium growth inhibition by peripheral blood mononuclear cells (PBMCs) is known to be one measure of protective immunity [14-17]. In the present study, we determined whether the magnitude of PPD responses by IFN- $\gamma$ assays or the TST was correlated with the subject's ability to kill intracellular mycobacteria, and examined this in relation to the subject's ESAT-6/CFP-10 responsiveness. We postulated that strong PPD responders without LTBI (ESAT-6/ CFP-10 nonresponders) might have distinctly different mycobacterium inhibition activity from LTBI subjects (ESAT-6/CFP10 responders), with similar PPD reactivity. This is because we believe PPD reactivity reflects protective immunity in the former group but not the latter.

The present study was performed in Singapore where there is moderate $\mathrm{TB}$ endemicity and routine BCG vaccination in newborns. Between the years 1958-2001, TST-nonresponding adolescents were revaccinated. Prevalent environmental mycobacteria exposure is probable, due to the equatorial climate [3]. There are therefore a number of reasons other than latent infection for strong PPD responsiveness in the Singapore adult population. We found that, when ESAT-6/CFP-10 responses were used to define LTBI, the correlation between the magnitude of PPD-specific IFN- $\gamma$ responses and mycobacterium inhibitory activity was diametrically opposite in LTBI and non-LTBI (uninfected) healthy subjects. This could underlie difficulties in attributing protective outcomes consistently with the magnitude of PPD responses.

\section{METHODS}

\section{Study subjects}

Our main study group comprised healthy household contacts ("contacts", $\mathrm{n=105)}$ of culture-proven TB patients at the Tuberculosis Control Unit (Tan Tock Seng Hospital, Singapore). The contacts had no physical or radiological signs of active or past TB disease and $73 \%$ had past BCG vaccination. The TST, using 1 tuberculin unit of PPD RT23 (Mantoux method), was read at 48-72 h. Contacts were recruited from among those offered chemoprophylaxis on clinical suspicion of LTBI, i.e. minimum criterion of TST $\geqslant 10 \mathrm{~mm}$ in those with one BCG scar and $\geqslant 16 \mathrm{~mm}$ for those with two BCG scars. These criteria were based on a previous local study relating TST size in schoolchildren and 4-yr risk of reactivation [18]. Chest radiograph cavities and smear positivity in the index case were also considered in offering prophylaxis. Analysis for tuberculin reactivity among TB contacts was adjusted for age, sex and BCG vaccination status, of which none were found to be sources of bias.
As comparator groups for the TB contacts, we also recruited 13 culture-positive pulmonary TB patients and 152 healthy community volunteers ("community"). The TB patients were PPD- and ESAT-6/CFP-10-reactive subjects within the first month of treatment. The community subjects were local residents attending a community general practice clinic for reasons unrelated to infectious or inflammatory disorders. Those with histories of past TB exposure were specifically excluded. Of these, $96 \%$ had at least one past BCG vaccination. Subjects aged $>85$ yrs or $<18$ yrs were excluded in all groups and the age distribution was comparable in all healthy groups. HIV infection was excluded on clinical history alone as the HIV rate among TB cases in Singapore is low. Informed consent for all volunteers was obtained by the attending physicians. Ethical review and approval of the study protocol was conducted by the Tan Tock Seng Hospital and National University of Singapore (Singapore) institutional review boards.

\section{Cell stimulation and IFN- $\gamma$ release assay}

IFN- $\gamma$ released by $2 \times 10^{5}$ viable PBMCs in response to $20 \mu \mathrm{g} \cdot \mathrm{mL}^{-1} \mathrm{PPD}$ or a mixture of 15 -mer overlapping synthetic peptides spanning the coding regions of ESAT- 6 and CFP-10 (published previously [19]) was measured in supernatants of 5day antigen-stimulated cultures by ELISA (OptEIA human IFN- $\gamma$; Becton Dickinson PharMingen, San Diego, CA, USA). The 5-day stimulation assay rather than overnight stimulation was used because it reflects memory response to the antigens [20] and minimises the risk of weak reactors being missed as prolonged incubation increases sensitivity for detecting LTBI [21]. IFN- $\gamma$ levels $4 \mathrm{SD}$ above the mean concentration in unstimulated control wells were regarded as "positive" based on published practice [19]. Positive ESAT-6/CFP-10 responders in contacts and community subjects were regarded in this study as having LTBI $[5,6]$, regardless of their TST size. Clinically healthy ESAT-6/CFP-10 nonresponders were deemed to have no LTBI.

\section{Mycobacterium inhibition assay}

The mycobacterium inhibition assay was modified from WORKU and HOFT [22]. Briefly, $2 \times 10^{5}$ viable PBMCs were seeded in RPMI 1640 (Sigma-Aldrich, St Louis, MO, USA) supplemented with $2 \mathrm{mM}$ L-glutamine (Sigma-Aldrich) and $10 \%$ fetal calf serum within 96-well plates. A sonicated preparation of dead M. tuberculosis ( $\mathrm{H} 37 \mathrm{Rv}$ strain, $1 \mu \mathrm{g} \cdot \mathrm{mL}^{-1}$ ) or PBS (control wells) was included in the cultures to expand mycobacterium-specific lymphocytes. After 2 days, $2 \times 10^{5}$ live M. bovis BCG (Pasteur strain) was added per well to infect the macrophages. Concurrently, supplementary ferric ammonium citrate $\left(50 \mu \mathrm{g} \cdot \mathrm{mL}^{-1}\right)$ was added for supporting optimal mycobacterium growth [23]. 3 days later, nonadherent cells were removed before infected macrophages were lysed with $0.1 \%$ saponin (Sigma-Aldrich) to release intracellular BCG. These released BCG were resuspended in Middlebrook 7H9 broth (Difco $^{\text {TM }}$ Middlebrook; Becton Dickinson, Sparks, NJ, USA), then pulsed with $1 \mu \mathrm{Ci}{ }^{3} \mathrm{H}$-uridine (Amersham Biosciences, Buckinghamshire, UK) for $48 \mathrm{~h}$ [24]. The radiolabelled BCG cultures were harvested onto membranes (Tomtec cell harvester; PerkinElmer Wallac, Boston, MA, USA) and radioactivity measured (Microbeta scintillation counter; PerkinElmer). The level of radioactivity expressed as counts per min (CPM) reflects 
the number of viable (actively replicating) bacteria in each well. Due to insufficient cell numbers for certain subjects after completion of various assays, only a subset of each study group was assessed in this assay (13 TB subjects, eight ESAT-6/CFP-10-responsive contacts, eight ESAT-6/CFP-10responsive community subjects, 27 ESAT-6/CFP-10-nonresponsive contacts and 17 ESAT-6/CFP-10-nonresponsive community subjects (total $n=73)$ ).

\section{Statistics}

The Kruskal-Wallis H-test was used for multigroup comparisons to determine whether the difference between groups was statistically significant. If this was significant, it was followed up with Dunn's multiple comparison post hoc test for pairwise comparison between two groups of interest. Spearman rank sum correlation and associated regression coefficients were used to analyse correlations between two independent variables. SPSS software version 16.0 (SPSS Inc., Chicago, IL, USA) was used for statistical computations.

\section{RESULTS}

\section{PPD, ESAT-6/CFP-10 and TST responses in different clinical groups}

Immune recognition of ESAT-6/CFP-10 and PPD was found in 18 and $72 \%$ of the contacts, respectively $(n=105)$ (table 1$)$. As these were "high risk" TB contacts with TST readings warranting prophylaxis for suspected LTBI, there was clearly discordance between the detection of LTBI by TST and ESAT-6/CFP-10 criteria. Response rates were 13 and 66\%, respectively, in the community group $(n=152)$. All ESAT-6/ CFP-10-responsive subjects responded to PPD.

Median TST responses were 16 and $14.5 \mathrm{~mm}$, respectively, in LTBI (ESAT-6/CFP-10-responsive) contacts and ESAT-6/CFP10-nonresponsive contacts (not shown); this difference was not statistically significant. Among all contacts, the correlation of TST size with PPD IFN- $\gamma$ responses, but not with ESAT-6/CFP-10
IFN- $\gamma$ responses, was significant $(r=0.21, p=0.03$ and $r=0.14$, $\mathrm{p}=0.16$, respectively; data not shown).

\section{Strong TST responders in ESAT-6/CFP-10-nonresponsive contacts show better in vitro mycobacterium inhibition than LTBI contacts}

Since a substantial proportion of contacts were strong TST reactors but ESAT-6/CFP-10-nonresponsive, we used the mycobacterium inhibition assay to study whether such discordant cases differed from the concordant (ESAT-6/CFP10 responders or LTBI) cases in killing activity.

First, we investigated how the magnitude of the TST response related to in vitro mycobacterium inhibition in contacts. Higher viable $B C G$ CPM were interpreted as signifying poorer mycobacterium inhibition activity. LTBI contacts with TST $\geqslant 16 \mathrm{~mm}$ had significantly poorer mycobacterium killing activity than the corresponding ESAT-6/CFP-10-nonresponsive contacts (fig. 1a). Indeed, the ESAT-6/CFP-10-nonresponsive group with the strongest TST responses $(\geqslant 16 \mathrm{~mm})$ showed the best in vitro mycobacterium inhibition, better than all LTBI groups. Among ESAT-6/CFP-10-nonresponsive contacts, the TST $\geqslant 16 \mathrm{~mm}$ group also showed better mycobacterium killing than the TST $10-15-\mathrm{mm}$ group. However, among those with LTBI there was no difference in mycobacterium inhibitory activity between subjects with different TST readings.

Although we knew that TST sizes correlated with the magnitude of in vitro PPD-specific IFN- $\gamma$ responses, it was not clear whether using the latter assay to determine PPD responsiveness (instead of the in vivo TST) would alter the relationship with mycobacterium killing. Additionally, we wanted to compare the contacts with the community subjects. Contacts and community subjects were therefore divided into PPDHigh and PPDLow groups based on IFN- $\gamma$ responses $\left(\geqslant 900 \mathrm{pg} \cdot \mathrm{mL}^{-1}\right.$ for PPDHigh, $<900 \mathrm{pg} \cdot \mathrm{mL}^{-1}$ for PPDLow) and mycobacterium inhibition was compared. The cut-off value was based on $70 \%$ of all LTBI subjects having at least this level

TABLE 1 In vitro responses to purified protein derivative (PPD)-based and early secreted antigenic target $6 \mathrm{kDa} / \mathrm{culture}$ filtrate protein 10 (ESAT-6/CFP-10)-based assays in different clinical groups

\begin{tabular}{|c|c|c|c|c|c|}
\hline \multirow[t]{2}{*}{ Group } & \multirow[t]{2}{*}{ Subjects n } & \multicolumn{2}{|c|}{ Positive tests $\#$} & \multicolumn{2}{|c|}{$\mathrm{TST}^{\circ}$} \\
\hline & & PPD & ESAT-6/CFP-10 & $10-15 \mathrm{~mm}$ & $\geqslant 16 \mathrm{~mm}$ \\
\hline Contacts & 105 & $76(72)$ & $19(18)$ & $69(66)$ & $36(34)$ \\
\hline LTBI contacts ${ }^{+}$ & 19 & $19(100)$ & $19(100)$ & $9(47)$ & $10(53)$ \\
\hline ESAT-6/CFP-10 nonresponsive contacts ${ }^{+}$ & 86 & $57(66)$ & $0(0)$ & $60(70)$ & $26(30)$ \\
\hline Community & 152 & $100(66)$ & $20(13)$ & & \\
\hline LTBI community $^{+}$ & 20 & $20(100)$ & $20(100)$ & & \\
\hline ESAT-6/CFP-10 nonresponsive community ${ }^{+}$ & 132 & $80(61)$ & $0(0)$ & & \\
\hline
\end{tabular}

Data are presented as n (\%), unless otherwise stated. TST: tuberculin skin test; LTBI: latent tuberculosis infection. ${ }^{\#}$ : the criteria for defining positive antigen-induced interferon- $\gamma$ response are given in the Methods section. ${ }^{\ddots}$ : TST was not performed for community subjects. Contacts with TST <10 mm were excluded from the study. The TST response was dichomotised at $16 \mathrm{~mm}$ based on a previous local study of schoolchildren which suggested that those with $\geqslant 16 \mathrm{~mm}$ TST readings had a higher risk of developing tuberculosis within 4 yrs [18]. + : LTBI subjects had positive responses to ESAT-6/CFP-10 and were regarded as having latent tuberculosis infection, ESAT-6/ CFP-10 nonresponding subjects were regarded as having no latent infection. 

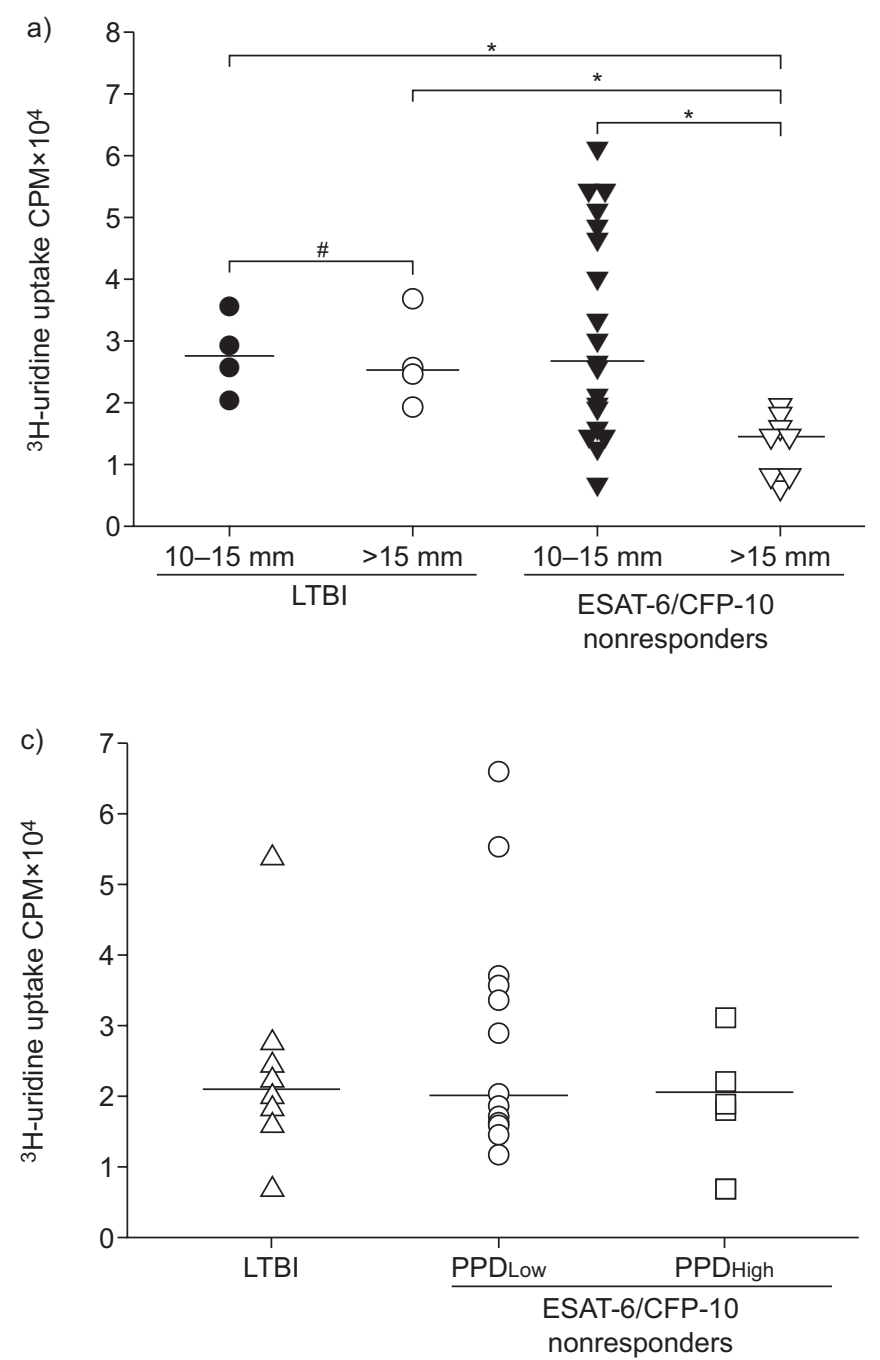

of response. Since IFN- $\gamma$ activates infected macrophages to kill intracellular M. tuberculosis [25], we hypothesised that the strength of the PPD-specific IFN- $\gamma$ responses might be positively associated with mycobacterium killing activity.

With this in vitro PPD IFN- $\gamma$ release assay (fig. $1 \mathrm{~b}$ ), the contacts showed a similar pattern of responses as with the TST; i.e. PPDHigh ESAT-6/CFP-10 nonresponders showed better mycobacterium inhibition than both the LTBI contacts and the PPDLow ESAT-6/CFP-10 nonresponders. The difference in mycobacterium killing between PPDHigh ESAT-6/CFP-10 nonresponders and LTBI subjects was not due to differential PPD IFN- $\gamma$ responses as these two groups had similar median IFN- $\gamma$ responses ( $p=0.67$; see fig. $1 \mathrm{a}$ of the online supplementary material). In contrast, the community subjects showed no differential mycobacterium killing activity based on PPD IFN- $\gamma$ reactivity (see fig. $1 \mathrm{~b}$ of the online supplementary material) or LTBI status (fig. 1c).

As an association was found between TST and killing activity in the contacts (fig. 1a), we studied the correlation between these parameters. In the ESAT-6/CFP-10-nonresponsive group the two parameters were significantly correlated: the larger the b)

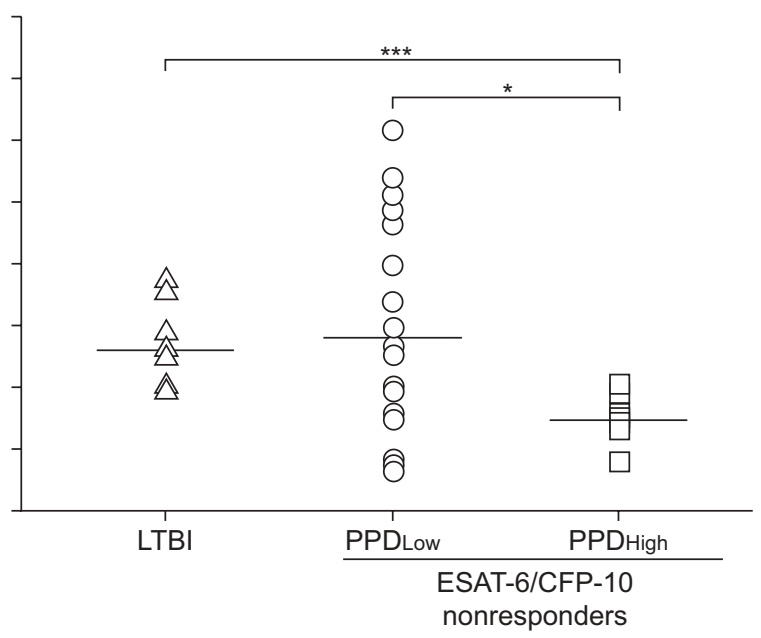

FIGURE 1. Mycobacterium inhibition in tuberculosis (TB) contacts and/or community subjects according to tuberculin skin test (TST) and in vitro purified protein derivative (PPD) interferon (IFN)- $\gamma$ responses. ${ }^{3} \mathrm{H}$-uridine uptake was used as a measure of mycobacterium viability, with higher counts per min (CPM) indicating more viable bacteria. a) TB contacts segregated according to TST size. Those with positive and negative responses to early secreted antigenic target $6 \mathrm{kDa}$ /culture filtrate protein-10 (ESAT-6/CFP-10) are identified as the latent TB infection (LTBI) group and ESAT-6/CFP-10 nonresponders, respectively. PPDHigh and PPDLow groups among b) TB contacts and c) community subjects had PPD-stimulated IFN- $\gamma \geqslant 900 \mathrm{pg} \cdot \mathrm{mL}^{-1}$ and $<900 \mathrm{pg} \cdot \mathrm{mL}^{-1}$, respectively. Bars represent group medians. Multigroup comparisons using Kruskal-Wallis $\mathrm{H}$-test showed statistically significant differences between groups in a) $p=0.012$ and b) $p=0.014$; thereafter the Dunn's multiple comparison post hoc test was performed. ${ }^{*}: p<0.05$; $* * *: p<0.001 ;{ }^{*}: p=0.67$.

TST size, the better the mycobacterium inhibition (fig. 2a). There was no correlation in the LTBI contacts group (fig. $2 b$ ).

\section{Stronger PPD-specific IFN- $\gamma$ responses correlated with better mycobacterium inhibition activity in ESAT-6/CFP-10 nonresponders but opposite correlation found in LTBI and TB subjects}

We hypothesised that, in uninfected persons, mycobactericidal activity should increase progressively with increases in PPD reactivity (if the latter is a marker of protective antimycobacterium immunity). Conversely, the magnitude of PPD reactivity in infected persons might not correlate with killing activity. This would explain previous observations that PPD responses are not always linked to protection [13]. We therefore separately assessed infected persons, including latent and active TB groups (healthy ESAT-6/CFP-10 responders and TB patients) versus uninfected persons (ESAT-6/CFP-10 nonresponders in both contacts and community groups). All subjects across the entire spectrum of PPD responses for both the infected and uninfected groups were included as we did not wish to make any assumptions regarding the impact of any specific range of PPD values on the correlation trend. 

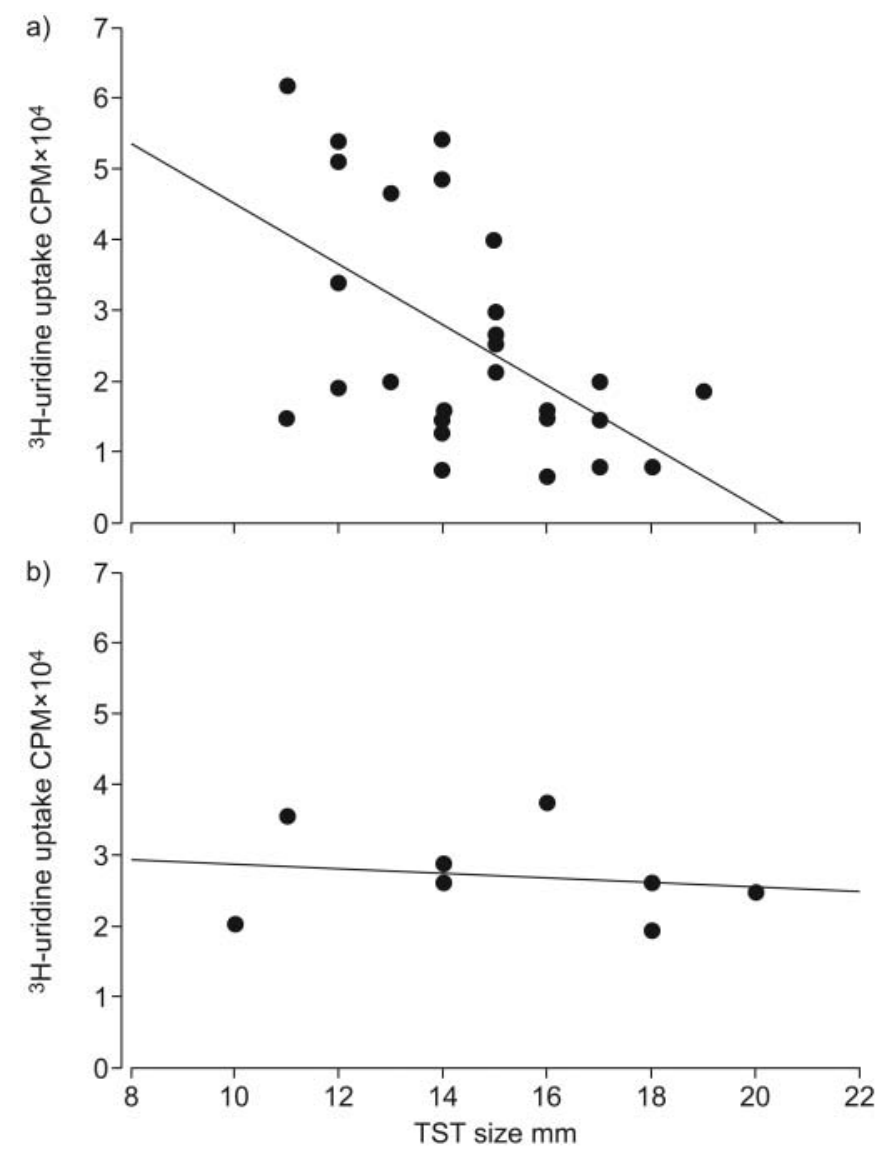

FIGURE 2. Correlation of tuberculin skin test (TST) outcomes in relation to mycobacterium inhibition in tuberculosis (TB) contacts. Regression lines and coefficients for correlations between TST sizes and counts per minute (CPM) ${ }^{3} \mathrm{H}$-uridine uptake for a) early secreted antigenic target $6 \mathrm{kDa}$ /culture filtrate protein 10 (ESAT-6/CFP-10) nonresponder contacts $(n=27)$ and b) latent TB infection contacts $(n=8)$. a) $r=-0.5, p=0.01 ; b) r=-0.27, p=0.53$ (Spearman rank sum correlation test).

There was an interesting dichotomy in the correlation graphs. Among ESAT-6/CFP-10 nonresponders, the higher the PPD IFN- $\gamma$ responses, the better the mycobacterium killing activity (fig. 3a). Conversely, among infected subjects, the higher the PPD IFN- $\gamma$ levels, the poorer the mycobacterium inhibition (fig. 3b). Both correlations were significant but the trends were opposite. Therefore, stronger PPD-specific IFN- $\gamma$ responses were associated with better mycobacterium killing only in subjects without latent or active infection.

\section{DISCUSSION}

In our study, of the subjects with moderate TB endemicity, only $18 \%$ of the healthy TST-reactive TB contacts responded to ESAT-6/CFP-10 (table 1). This discordance is probably due to prior BCG vaccination and/or environmental mycobacteria exposure priming TST responses in those without TB exposure. The TB contacts in our study had an average age of $40 \mathrm{yrs}$. The extent of sensitisation with environmental mycobacteria is known to increase with age [26], and TST readings are strongly influenced by environmental mycobacteria exposure [27]. Moreover, BCG priming in childhood may potentially be boosted by repeated exposure to $M$. tuberculosis in Singapore where the incidence of $\mathrm{TB}$ is moderately high.

Two main points were shown by our data. First, strongly PPDreactive ESAT-6/CFP-10 nonresponders in our population had good antimycobacterium immunity. They not only showed better mycobacterium killing than the ESAT-6/CFP-10 nonresponders with lower PPD reactivity (fig. $1 \mathrm{a}$ and $\mathrm{b}$ ), but the magnitude of PPD reactivity was also correlated with killing (fig. 2). These findings support the probability that, in those without latent infection in our study population, PPD reactivity reflects the strength of antimycobacterium immunity in each person, consistent with a previous report that tuberculinpositive subjects show better inhibition of mycobacterial growth than tuberculin-negative individuals [17]. Secondly, our strongly PPD-reactive ESAT-6/CFP-10-nonresponsive contacts had distinct antimycobacterium immunity from LTBI contacts as the former had better mycobacterium inhibition activity
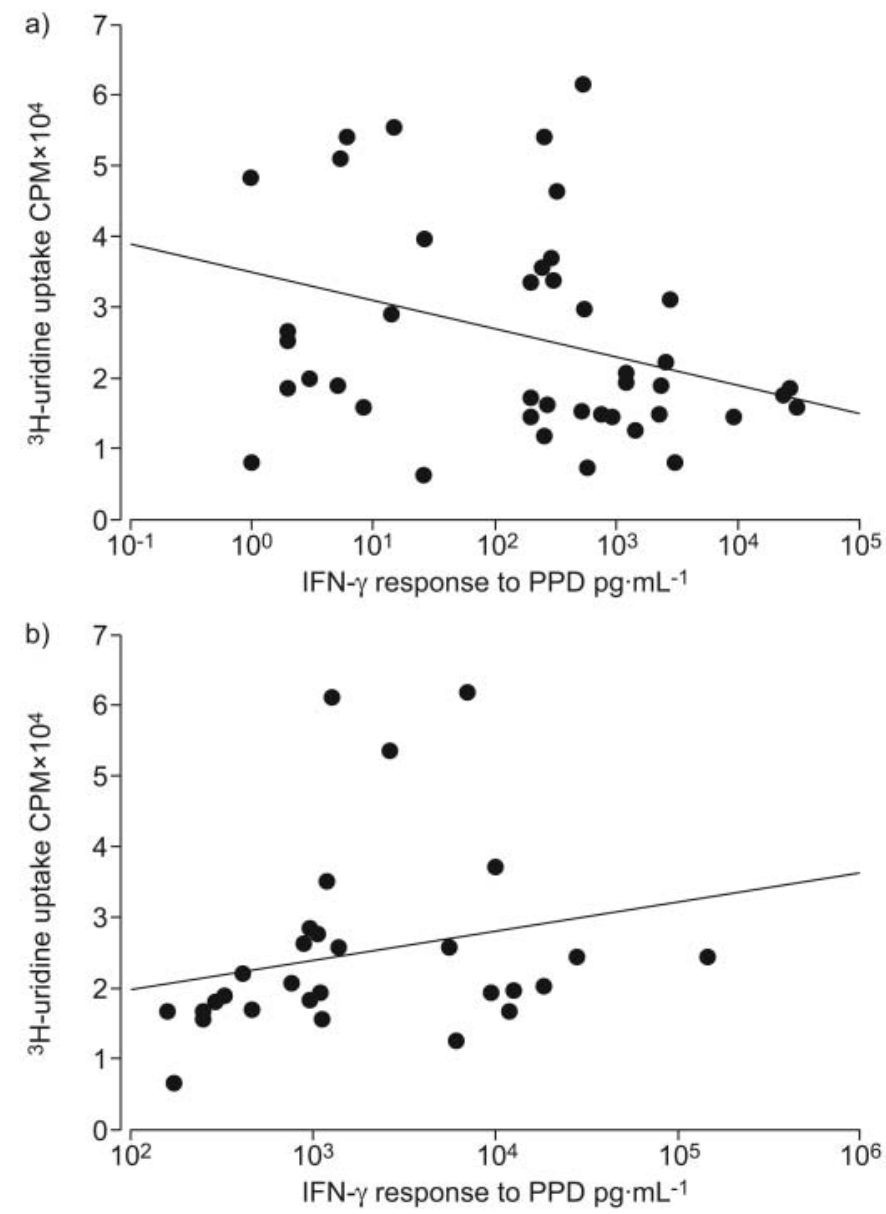

FIGURE 3. Opposite correlation trends between mycobacterium inhibition and purified protein derivative (PPD) interferon (IFN)- $\gamma$ response in a) the uninfected group (early secreted antigenic target $6 \mathrm{kDa} /$ culture filtrate protein 10 nonresponders, $n=44$ ) and $b$ ) the infected group (tuberculosis (TB) and latent TB infection subjects, $n=29$ ). Both groups included contacts and community subjects Regression lines and coefficients are shown. a) $r=-0.32, p=0.03$; b) $r=0.4$, $\mathrm{p}=0.03$ (Spearman rank sum correlation test). CPM: counts per minute. 
(fig. $1 \mathrm{a}$ and b). There were also opposite trends in the association between PPD reactivity and mycobacterium killing in infected and uninfected groups (fig. 3). We acknowledge, however, that the sample of LTBI contacts studied in figure 3 was small $(n=8)$. Nonetheless, taken together, we believe that in communities with other known sources of mycobacterium antigen priming besides LTBI, strongly PPD-reactive ESAT-6/ CFP-10 nonresponders should not generally be regarded as having LTBI and could even have some protective immunity [2]. In stating this, we have assumed that inhibition of mycobacterium survival is a marker of protective immunity. We acknowledge that there is no direct prospective epidemiological evidence that this assay predicts long-term protection.

However, a number of published studies support this assumption. Using a mycobacterium inhibition assay, SILVER et al. [28] showed an association between in vitro lymphocyte responses and inhibition of intracellular M. tuberculosis growth; the assay therefore reflected the contribution of mycobacteria-specific Tcells to protection. HoFT and co-workers [15, 16] found that inhibition of intracellular M. tuberculosis replication significantly increased after BCG vaccination of initially PPD-nonreactive persons, thus reflecting BCG-induced protective immunity. Taken together with evidence that tuberculin-positive persons can restrict growth of BCG better than those who are tuberculinnegative [17], these studies demonstrate that assays of BCG killing by blood immune cells reflect the host's mycobacteriumspecific immunity, not just innate immune mechanisms. Moreover, we and others [22] have shown that the assay is specific for mycobacterium-reactive T-cells (see fig. 2 of the online supplementary material) by demonstrating that unstimulated T-cells or cells specific for non-mycobacterium antigens have weaker killing activity than mycobacterium antigenspecific cells. The utility of the mycobacterium growth inhibition assay in reflecting protective immunity has led to its recent use as a surrogate marker for vaccine-induced protection in a recombinant BCG vaccine study [14].

It is possible that $M$. tuberculosis sonicate stimulation of lymphocytes from LTBI cases may result in expansion of ESAT-specific lymphocytes that do not recognise BCG-infected cells. This may theoretically be one contributory factor to the poorer killing activity of LTBI relative to ESAT-6/CFP-10 nonresponders. However, with our lymphocyte cultures exposed to both whole $M$. tuberculosis sonicate and live BCG, there is likely to be considerable expansion of T-cells specific for the many immunodominant antigens shared between BCG and $M$. tuberculosis, and such cells are known to contribute to mycobacterium killing [29].

When different types of mycobacterium inhibition assays were conducted on PPD-negative persons given BCG immunisation, subsequent BCG-specific IFN- $\gamma$ production was not correlated with any of the assays [15]. If PPD-positive subjects in our study were taken together, there would also be an apparent lack of correlation of PPD-specific IFN- $\gamma$ responses with mycobacterium killing because there are opposite correlation trends in ESAT-6/CFP-10 nonresponders and those with LTBI (fig. 3). Some possible explanations for this paradox follow.

TB-specific IFN- $\gamma$ production activates macrophages to inhibit intracellular mycobacterium growth [30] but, under some circumstances, IFN- $\gamma$ promotes intracellular mycobacterium replication [28] and contributes to apoptosis of $M$. tuberculosisresponsive T-cells, thus favouring $M$. tuberculosis persistence [31]. This may happen in infected persons. For those with LTBI, it is also possible that stronger PPD reactivity reflects subjects with higher bacterial loads. Alternatively, regulatory T-cells in infected persons may modulate the ability of antigen-specific T-cells to kill mycobacteria. In active TB and LTBI, increased frequency of peripheral blood CD4+CD25+ FoxP3+ T-cells (putative regulatory T-cells) has been associated with suppressed $M$. tuberculosis-specific immunity [32-34]. In late-stage murine $\mathrm{TB}$, transforming growth factor- $\beta$, a suppressive cytokine associated with regulatory T-cells, impairs the host's ability to limit M. tuberculosis survival [35]. These are some potential reasons why PPD-specific IFN- $\gamma$ responses were dissociated from mycobacterium inhibition in infected subjects.

In vitro ESAT-6/CFP-10-based tests for diagnosing LTBI are gaining clinical acceptance [36] and discordance with traditional TST readings needs to be resolved for decisions on prophylaxis. In the Singapore population, the discordance in TST and ESAT-6/CFP-10 responses is largely unidirectional; cases of TST $<10 \mathrm{~mm}$ comprised only $4 \%$ of ESAT-6/CFP-10responsive $\mathrm{TB}$ contacts completing prophylactic treatment in a previous local study [37], whereas ESAT-6/CFP-10nonresponsive cases constituted $>80 \%$ of our TST-positive contacts (table 1). With regard to stratifying risk in TB contacts, in our epidemiological setting we have shown that the magnitude of the TST responses may not consistently reflect protective outcomes. The good correlation between mycobacterium killing activity and PPD responses in the ESAT-6/CFP10-nonresponsive contacts supports the probability that strong PPD reactors in this group are unlikely to be at high risk. Conversely, strong PPD reactors in ESAT-6/CFP-10-positive contacts are likely to warrant closer follow-up as they have relatively weaker mycobacterium killing activity. Such a distinction between PPD-reactive contacts could help to channel healthcare resources based on risk. However, we emphasise that this strategy may only be applicable in a setting of moderate to high TB endemicity where there are obvious reasons for strong PPD reactivity other than LTBI. Our results also suggest that it is relevant to assess the latent infection rate in trial populations prior to TB vaccine studies as we found an association between strong PPD reactivity and poorer mycobacterium inhibition in LTBI subjects. Therefore, if LTBI is highly prevalent in the trial population and vaccines strongly inducing PPD reactivity are favoured, this may paradoxically lead researchers to choose vaccines that give rise to relatively poorer mycobacterium inhibition.

\section{SUPPORT STATEMENT}

This study was funded by the Ministry of Education, Singapore (AcRF Tier 2 grant no. R182000085112).

\section{STATEMENT OF INTEREST}

None declared.

\section{ACKNOWLEDGEMENTS}

The authors gratefully acknowledge phlebotomy support from nursing staff at the TB Control Unit, Tan Tock Seng Hospital (Singapore). 


\section{REFERENCES}

1 Menzies R, Vissandjee B. Effect of bacille Calmette-Guerin vaccination on tuberculin reactivity. Am Rev Respir Dis 1992; 145: 621-625.

2 Black GF, Weir RE, Floyd S, et al. BCG-induced increase in interferon- $\gamma$ response to mycobacterial antigens and efficacy of BCG vaccination in Malawi and the UK: two randomised controlled studies. Lancet 2002; 359: 1393-1401.

3 Fine PE. Variation in protection by BCG: implications of and for heterologous immunity. Lancet 1995; 346: 1339-1345.

4 Behr MA, Wilson MA, Gill WP, et al. Comparative genomics of BCG vaccines by whole-genome DNA microarray. Science 1999; 284: 1520-1523.

5 Lalvani A. Diagnosing tuberculosis infection in the 21st century: new tools to tackle an old enemy. Chest 2007; 131: 1898-1906.

6 Pai M, Riley LW, Colford JM Jr. Interferon- $\gamma$ assays in the immunodiagnosis of tuberculosis: a systematic review. Lancet Infect Dis 2004; 4: 761-776.

7 Lalvani A, Pathan AA, Durkan H, et al. Enhanced contact tracing and spatial tracking of Mycobacterium tuberculosis infection by enumeration of antigen-specific T cells. Lancet 2001; 357: 2017-2021.

8 Doherty TM, Demissie A, Olobo J, et al. Immune responses to the Mycobacterium tuberculosis-specific antigen ESAT-6 signal subclinical infection among contacts of tuberculosis patients. J Clin Microbiol 2002; 40: 704-706.

9 Hill PC, Brookes RH, Fox A, et al. Large-scale evaluation of enzyme-linked immunospot assay and skin test for diagnosis of Mycobacterium tuberculosis infection against a gradient of exposure in The Gambia. Clin Infect Dis 2004; 38: 966-973.

10 Dosanjh DP, Hinks TS, Innes JA, et al. Improved diagnostic evaluation of suspected tuberculosis. Ann Intern Med 2008; 148: 325-336.

11 Mazurek GH, Weis SE, Moonan PK, et al. Prospective comparison of the tuberculin skin test and 2 whole-blood interferon- $\gamma$ release assays in persons with suspected tuberculosis. Clin Infect Dis 2007; 45: 837-845.

12 Ottenhoff TH, Kumararatne D, Casanova JL. Novel human immunodeficiencies reveal the essential role of type-I cytokines in immunity to intracellular bacteria. Immunol Today 1998; 19: 491-494.

13 Wassie L, Demissie A, Aseffa A, et al. Ex vivo cytokine mRNA levels correlate with changing clinical status of Ethiopian TB patients and their contacts over time. PLoS One 2008; 3: e1522.

14 Hoft DF, Blazevic A, Abate G, et al. A new recombinant bacille Calmette-Guerin vaccine safely induces significantly enhanced tuberculosis-specific immunity in human volunteers. J Infect Dis 2008; 198: 1491-1501.

15 Hoft DF, Worku S, Kampmann B, et al. Investigation of the relationships between immune-mediated inhibition of mycobacterial growth and other potential surrogate markers of protective Mycobacterium tuberculosis immunity. J Infect Dis 2002; 186: $1448-1457$.

16 Worku S, Hoft DF. Differential effects of control and antigenspecific T cells on intracellular mycobacterial growth. Infect Immun 2003; 71: 1763-1773.

17 Kampmann B, Gaora PO, Snewin VA, et al. Evaluation of human antimycobacterial immunity using recombinant reporter mycobacteria. J Infect Dis 2000; 182: 895-901.

18 Chee $\mathrm{CB}$, Soh $\mathrm{CH}$, Boudville IC, et al. Interpretation of the tuberculin skin test in Mycobacterium bovis BCG-vaccinated Singaporean schoolchildren. Am J Respir Crit Care Med 2001; 164: 958-961.

19 Arend SM, Geluk A, van Meijgaarden KE, et al. Antigenic equivalence of human T-cell responses to Mycobacterium tuberculosis-specific RD1-encoded protein antigens ESAT-6 and culture filtrate protein 10 and to mixtures of synthetic peptides. Infect Immun 2000; 68: 3314-3321.

20 Ferrand RA, Bothamley GH, Whelan A, et al. Interferon- $\gamma$ responses to ESAT-6 in tuberculosis patients early into and after antituberculosis treatment. Int J Tuberc Lung Dis 2005; 9: 1034-1039.
21 Leyten EM, Arend SM, Prins C, et al. Discrepancy between Mycobacterium tuberculosis-specific $\gamma$ interferon release assays using short and prolonged in vitro incubation. Clin Vaccine Immunol 2007; 14: 880-885.

22 Worku S, Hoft DF. In vitro measurement of protective mycobacterial immunity: antigen-specific expansion of $\mathrm{T}$ cells capable of inhibiting intracellular growth of bacille Calmette-Guerin. Clin Infect Dis 2000; 30: Suppl. 3, S257-S261.

23 Rook GA, Steele J, Ainsworth M, et al. Activation of macrophages to inhibit proliferation of Mycobacterium tuberculosis: comparison of the effects of recombinant $\gamma$-interferon on human monocytes and murine peritoneal macrophages. Immunology 1986; 59: 333-338.

24 Fazal N, Bartlett R, Lammas DA, et al. A comparison of the different methods available for determining BCG-macrophage interactions in vitro, including a new method of colony counting in broth. FEMS Microbiol Immunol 1992; 5: 355-362.

25 Flesch I, Kaufmann SH. Mycobacterial growth inhibition by interferon- $\gamma$-activated bone marrow macrophages and differential susceptibility among strains of Mycobacterium tuberculosis. J Immunol 1987; 138: 4408-4413.

26 Black GF, Fine PEM, Warndorff DK, et al. Relationship between IFN- $\gamma$ and skin test responsiveness to Mycobacterium tuberculosis PPD in healthy, non-BCG-vaccinated young adults in Northern Malawi. Int J Tuberc Lung Dis 2001; 5: 664-672.

27 Black GF, Dockrell HM, Crampin AC, et al. Patterns and implications of naturally acquired immune responses to environmental and tuberculous mycobacterial antigens in northern Malawi. J Infect Dis 2001; 184: 322-329.

28 Silver RF, Li Q, Boom WH, et al. Lymphocyte-dependent inhibition of growth of virulent Mycobacterium tuberculosis H37Rv within human monocytes: requirement for $\mathrm{CD} 4+\mathrm{T}$ cells in purified protein derivative-positive, but not in purified protein derivativenegative subjects. J Immunol 1998; 160: 2408-2417.

29 Geluk A, van Meijgaarden KE, Franken KL, et al. Identification of major epitopes of Mycobacterium tuberculosis AG85B that are recognized by HLA-A*0201-restricted CD8+ T cells in HLAtransgenic mice and humans. J Immunol 2000; 165: 6463-6471.

30 Flesch IE, Kaufmann SH. Activation of tuberculostatic macrophage functions by $\gamma$ interferon, interleukin-4, and tumor necrosis factor. Infect Immun 1990; 58: 2675-2677.

31 Hirsch CS, Toossi Z, Johnson JL, et al. Augmentation of apoptosis and interferon- $\gamma$ production at sites of active Mycobacterium tuberculosis infection in human tuberculosis. J Infect Dis 2001; 183: 779-788.

32 Hougardy JM, Place S, Hildebrand M, et al. Regulatory T cells depress immune responses to protective antigens in active tuberculosis. Am J Respir Crit Care Med 2007; 176: 409-416.

33 Hougardy JM, Verscheure $\mathrm{V}$, Locht $\mathrm{C}$, et al. In vitro expansion of CD4+CD25highFOXP3+CD127low/- regulatory T cells from peripheral blood lymphocytes of healthy Mycobacterium tuberculosisinfected humans. Microbes Infect 2007; 9: 1325-1332.

34 Chiacchio $\mathrm{T}$, Casetti $\mathrm{R}$, Butera $\mathrm{O}$, et al. Characterization of regulatory $\mathrm{T}$ cells identified as CD4(+)CD25(high)CD39(+) in patients with active tuberculosis. Clin Exp Immunol 2009; 156: 463-470.

35 Hernandez-Pando R, Orozco-Esteves H, Maldonado HA, et al. A combination of a transforming growth factor-beta antagonist and an inhibitor of cyclooxygenase is an effective treatment for murine pulmonary tuberculosis. Clin Exp Immunol 2006; 144: 264-272.

36 Mazurek GH, Villarino ME. Guidelines for using the QuantiFERON-TB test for diagnosing latent Mycobacterium tuberculosis infection. Centers for Disease Control and Prevention. MMWR Recomm Rep 2003; 52: 15-18.

37 Chee CB, KhinMar KW, Gan $\mathrm{SH}$, et al. Latent tuberculosis infection treatment and $\mathrm{T}$-cell responses to Mycobacterium tuberculosis-specific antigens. Am J Respir Crit Care Med 2007; 175: 282-287. 